\title{
The ground-based counterpart of the CoRoT asteroseismic observations from space
}

\author{
K. Uytterhoeven, ${ }^{1}$ E. Poretti ${ }^{1}$ and the COROT SGBOWG \\ ${ }^{1}$ INAF-Osservatorio Astronomico di Brera, Merate (LC), Italy
}

The CoRoT mission (Michel et al. 2006, 2007) needs a significant amount of ground-based observing time to achieve its science goals. Ground-based asteroseismology has progressed under several aspects (from single- to multi-site campaigns, closer matching between observed frequencies and mode identification, tighter collaboration between observers and theoreticians, etc.). The scientific cases of the $\delta$ Sct variable FG Vir (more than 75 frequencies identified; Breger et al. 2005) and of the $\beta$ Cep variable $\nu$ Eri (Ausseloos et al. 2004; De Ridder et al. 2004) can be considered as the frontiers of the ground-based techniques. A large observational effort has been made in the past decades to extract sets of frequencies from photometry (light and colour data) and spectroscopy (line-profile and radial-velocity variations), often combined together. Now, observations from space constitute the natural development of all the progress made by the asteroseismic community, both in the development of methods and of techniques and in the description of the stellar interiors. CoRoT is expected to introduce a dramatic increase in the number of identified frequencies.

CoRoT targets cover the whole Main Sequence with the goal to monitor a broad variety of pulsational and other variability mechanisms. For the pulsators, the CoRoT photometry will give us access to low-degree modes, at an amplitude level three orders of magnitude smaller than the current one. However, the complete frequency spectrum is necessary to investigate the stellar interior, including rotation. Therefore, we need to accompany the CoRoT photometric time series with spectroscopic observations, since, in spectra with a resolution $\sim 50000$, modes with degrees as high as $\ell=10$ can be detected through the analysis of the line-profile variations. In moderate and fast rotators, spectral resolution translates into spatial resolution by disentangling different stripes of the stellar surface. As a result, non-radial modes produce line-profile perturbations visible as "moving bumps" in the line profiles. A detailed analysis of the line-profile variations allows us to identify corresponding modes because the shape and the behaviour of the variations depend on the properties of the modes. Indeed, such line-profile analyses have been successfully carried out for several classes of non-radially pulsating stars.

To combine the photometric and spectroscopic approaches, we applied to obtain telescope time at different observatories. In particular, a Large Programme has been granted at the FEROS@2.2m ESO-MPI instrument, focused on the $\delta \mathrm{Sct}, \gamma$ Dor, $\beta$ Cep and Be stars among the CoRoT targets. Sixty nights (15 in each semester, split into two strings of 10 and 5 consecutive nights separated by at least 10 days) will be devoted to the CoRoT programme in four consecutive ESO Periods, covering the first 1.5 years of the CoRoT lifetime. FEROS is a very solid baseline for the observations of $\delta$ Sct, $\gamma$ Dor, $\beta$ Cep and Be stars, having been used in the past (e.g., Zima et al. 2006). Moreover, other Large Programmes will be carried out at the Observatoire de Haute Provence (using the new SOPHIE@1.92m instrument; PI P. Mathias) and at the Calar Alto Observatory (using the FOCES@2.2m instrument; PI P. Amado). Normal proposals on specific targets will be submitted to the Nordic Optical Telescope (FIES and SOFIN instruments) and Telescopio Nazionale Galileo (SARG instrument) to supply complementary data to those of the ESO, OHP and Calar Alto Large Programs.

Therefore, we feel ready to accompany the CoRoT mission with the best observational ground-based effort, in order that as much information as possible can be extracted from this huge (scientific, financial and manpower) effort that the astronomical community is undertaking. 
Acknowledgments. The Seismology Ground-Based Observations Working Group (SGBOWG) involves a wide participation and know-how from researchers in different Institutes: A. Baglin, C. Catala, E. Michel, M. Floquet, M. J. Goupil, A. M. Hubert, Y. Lebreton, A. Moya, C. Neiner, (Meudon Observatory), C. Aerts, M. Briquet, M. Desmet, W. Zima (Leuven University), P. Amado, R. Garrido, S. Martín-Ruiz, J. C. Suárez (IAA Granada), L. Mantegazza, E. Poretti, M. Rainer, K. Uytterhoeven (INAF-OABrera), P. Mathias (OCA Nice) and F. X. Schmider (Nice University). K. Uytterhoeven acknowledges the support of the European Community under the Marie Curie Intra-European Fellowship, Contract 024476PrepCOROT.

\section{References}

Ausseloos M., Scuflaire R., Thoul A., Aerts C., 2004, MNRAS, 355, 352

Breger M., Lenz P., Antoci V., et al., 2005, A\&A, 435, 955

De Ridder J., Telting J. H., Balona L. A., et al., 2004, MNRAS, 351, 324

Michel E., Samadi R., Baudin F., et al., 2006, Mem. Soc. Astron. It., 77, 539

Michel E., Baglin A., Samadi R., Baudin F., Auvergne M., 2007, Comm. Asteroseis., 150, 341

Zima W., Wright D., Bentley J., et al., 2006, A\&A, 455, 235 